\title{
Diversité et gestion durable des Loranthaceae dans les hautes terres de l'Ouest du Cameroun
}

René Bernardin JIOFACK TAFOKOU ${ }^{1}$ Jean Paul Dondjang ${ }^{2}$

Bernard-Aloys NKONGMENECK ${ }^{1}$

M. SMITH ${ }^{3}$

Victor KemeuzE ${ }^{1}$

${ }^{1}$ Ecologic Museum of Cameroon BP 8038

Yaoundé

Cameroun

2 Université de Dschang Faculté d'agronomie et des sciences agricoles Département de foresterie BP 222

Dschang

Cameroon

${ }^{3}$ Natural History Museum Department of Botany Cromwell Road Londres SW7 5BD Royaume-Uni

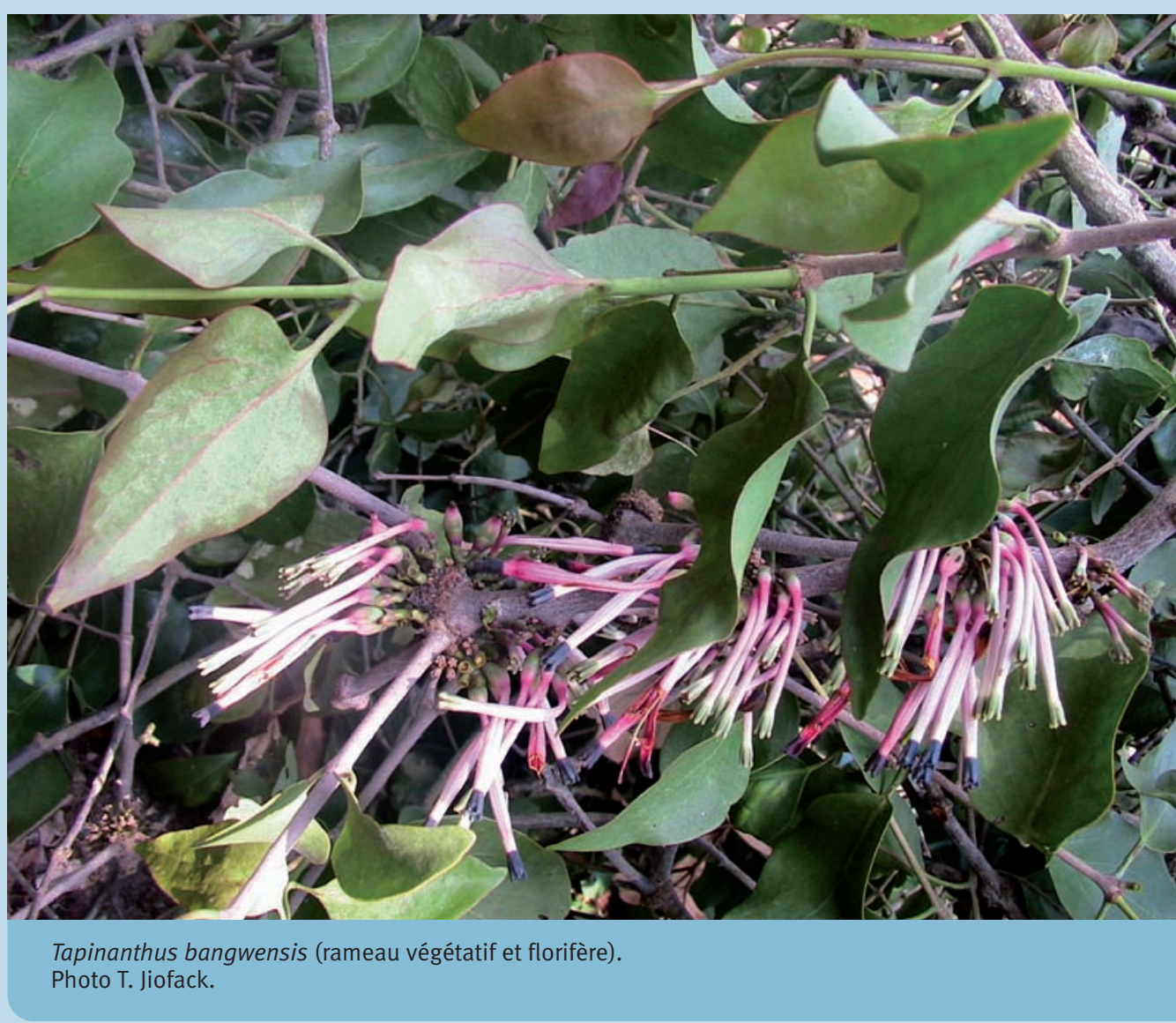




\section{RÉSUMÉ}

\section{DIVERSITÉ ET GESTION DURABLE DES LORANTHACEAE DANS LES HAUTES TERRES DE L'OUEST DU CAMEROUN}

Dans les hautes terres de l'Ouest du Cameroun, l'étude est centrée sur la présence et la diversité des espèces de Loranthaceae dans neuf localités du groupement Bafou. Elle apporte une nouvelle contribution à la valorisation et à la gestion durable des ressources biologiques d'écosystèmes forestiers profondément dégradés dans le bassin du Congo. L'étude a été réalisée au moyen d'observations de terrain et en recourant à la méthode active de recherche et de planification participative (Marpp). Elle repose essentiellement sur des interviews et des enquêtes semi-structurées individuelles conduites auprès des populations locales, paysans agriculteurs et tradithérapeutes, tous supposés détenir des connaissances sur la valorisation traditionnelle des Loranthaceae. Les enquêtes ont permis de recenser six espèces de Loranthaceae parasites de neuf espèces de fruitiers appartenant à sept familles végétales. Ces mêmes espèces, communément regroupées sous l'appellation locale de gui, font partie intégrante de la pharmacopée traditionnelle et sont utilisées sous la forme de plusieurs potions et/ou lotions, soit isolément, soit en association avec d'autres plantes ou d'autres drogues pour soigner plus d'une vingtaine de pathologies. Au terme de ce travail, des approches de gestion durable ont été proposées en remplacement des coupes trop destructrices sur hôtes pratiquées par les paysans. Ce système de contrôle, courant dans la région, compromet la régénération et la productivité des arbres fruitiers en particulier et la diversité des populations d’arbres en général.

Mots-clés : Loranthaceae, pharmacopée traditionnelle, tradithérapeute, groupement Bafou, gestion durable.

\section{ABSTRACT}

\section{DIVERSITY AND SUSTAINABLE MANAGEMENT OF LORANTHACEAE IN THE UPLANDS OF WEST CAMEROON}

This study in the uplands of Western Cameroon focused on the presence and diversity of Loranthaceae species in nine localities in the Bafou district. It offers a new contribution in the field of sustainable management and use of biological resources in severely degraded forest ecosystems in the Congo Basin. The study was based on the active method of research and participatory planning (ARMP). It relied essentially on surveys and individual semi-structured interviews with local people, peasant farmers and traditional healers, all of whom are reputedly knowledgeable on traditional uses of Loranthaceae. Six Loranthaceae were found during the surveys, growing as parasites on nine fruit tree species belonging to seven different plant families. These six species, commonly known as "mistletoe" among local people, are an integral part of the traditional pharmacopoeia and are used in the form of different potions and/or lotions, either on their own or in association with other plants or medicines, to treat some twenty different pathologies. The study concludes with proposals for sustainable management approaches to replace the local over-destructive practice of lopping from host trees. This method of controlling parasitic plants is common in the region but compromises regeneration and productivity, especially in fruit trees, and the diversity of tree populations in general.

Keywords: Loranthaceae, traditional pharmacopoeia, traditional healer, Bafou district, sustainable management.

\section{RESUMEN}

\section{DIVERSIDAD Y MANEJO SOSTENIBLE DE LAS LORANTÁCEAS EN LAS TIERRAS ALTAS DE CAMERÚN OCCIDENTAL}

Realizado en las tierras altas del oeste de Camerún, este estudio se centra en la presencia y diversidad de especies de lorantáceas en nueve localidades de la agrupación Bafou. Este trabajo aporta una nueva contribución al aprovechamiento y manejo sostenible de los recursos biológicos de ecosistemas forestales profundamente degradados en la cuenca del Congo. El estudio se efectuó mediante observaciones de campo y con la utilización del método activo de investigación y planificación participativa (MARPP, por sus siglas en francés). Está basado fundamentalmente en entrevistas y encuestas semiestructuradas individuales realizadas entre la población local: campesinos agricultores y terapeutas tradicionales; es decir, aquellos que supuestamente poseen los conocimientos sobre el aprovechamiento tradicional de las lorantáceas. Las encuestas permitieron inventariar seis especies de lorantáceas que parasitan nueve especies frutales pertenecientes a siete familias vegetales. Estas mismas especies, comúnmente agrupadas bajo la denominación local de muérdago, forman parte integrante de la farmacopea tradicional y se utilizan en forma de diferentes pociones o lociones, ya sea aisladas 0 en asociación con otras plantas o drogas, para curar más de veinte patologías distintas. Durante este trabajo se propusieron enfoques de manejo sostenible que sustituyan las cortas, excesivamente destructoras para los hospederos, que practican los campesinos. Este sistema de control, corriente en esta región, compromete la regeneración y productividad de los árboles frutales en particular y la diversidad de las poblaciones de árboles en general.

Palabras clave: lorantáceas, farmacopea tradicional, terapeuta tradicional, agrupación Bafou, manejo sostenible. 


\section{Introduction}

Les Loranthaceae, désignées sous le vocable commun de "gui d'Afrique ", sont des phanérogames, hémiparasites chlorophylliens ou épiphytes qui, implantés sur les parties aériennes de leurs hôtes, sont responsables de dégâts économiques, écologiques, morphogénétiques et technologiques variables selon les cultures ou les essences ligneuses parasitées (SAllé et al., 1998).

Dans les régions tempérées, cette famille végétale regroupe 950 espèces réparties en 77 genres. En Afrique et en Arabie, PolHILl et Wiens (1998) dénombrent plus de 500 espèces. Au Cameroun, BALLE (1986) recense sept genres réunissant près de 25 espèces.

Les hautes terres de l'Ouest du Cameroun constituent le grenier communautaire qui assure le ravitaillement d'une grande partie de la métropole en produits vivriers, notamment les villes de Yaoundé et de Douala (Dongmo et al., 1990). La problématique rurale majeure relève de la lutte contre la pauvreté et la sous-alimentation, en relation avec une démographie explosive qui est à l'origine de la disparition des recrûs forestiers. Cette situation est à l'origine des plantations agroforestières et des jardins de case, au sein desquels les arbres fruitiers sont très souvent attaqués par de nombreux phytoparasites. Ces derniers sont quelquefois mésestimés par les paysans, bien qu'à l'origine de nombreux dégâts sur leurs récoltes.

Ces hémiparasites sont des végétaux qui ont perdu au cours de leur évolution leur autonomie dans l'accès aux ressources nutritives et sont devenus tributaires de leurs hôtes. Ils représentent un fléau réel contre lequel une lutte volontariste s'avère indispensable, compte tenu de l'ampleur des dégâts. SALLÉ et ABER (1986) mentionnent qu'une telle lutte passe nécessairement par une meilleure connaissance de la répartition géographique et de la biologie des organismes ciblés.

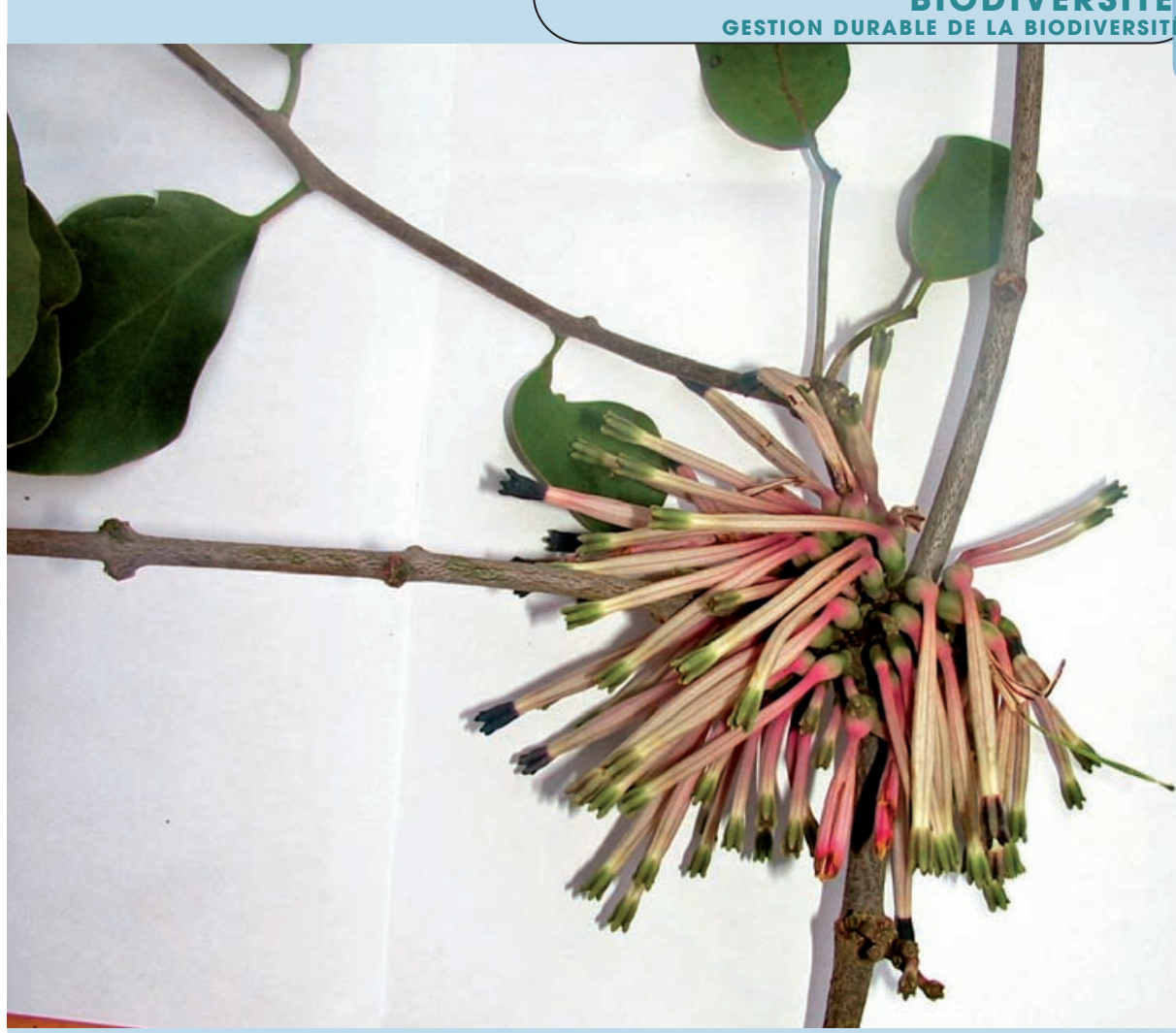

Tapinanthus bangwensis (inflorescence immature). Photo T. Jiofack.

Toutefois, les Loranthaceae présentent également de nombreuses vertus thérapeutiques. D'une région à l'autre, leur utilisation se manifeste soit de manière isolée, soit en fonction d'un hôte approprié ou spécifique, soit enfin en association avec d'autres drogues ou solvants (IIOFACK et al., 2007). Dès le XVII siècle, les herboristes prescrivent le gui européen contre l'épilepsie et les désordres nerveux, et le recommandent pour régulariser les activités glandulaires, le rythme cardiaque et la digestion (LIEUtAghI, 1991 ; Rodzko, 2000). La viscine, substance collante de couleur orangée qui recouvre les graines, ralentirait dangereusement le rythme cardiaque, causerait des convulsions, élèverait la pression artérielle et provoquerait l'avortement à forte dose (LIEUTAGHI, 1991 ; Rodzko, 2000). Ces mêmes auteurs ajoutent également qu'à faible dose des effets bénéfiques sont constatés sur des personnes souffrant d'hypertension artérielle et de maladies cardiaques. PolHILl et WIENS (1998) relèvent l'attribution d'effets "magiques » en relation directe avec le caractère répandu de l'utilisation médicinale des Loranthaceae. Ces plantes sont utilisées aussi bien dans des cas de sorcellerie que pour des problèmes urogénitaux, les courbatures, le rachitisme, la toux et les problèmes pulmonaires.

Le but de cette étude est de promouvoir la diversité spécifique et la vulgarisation du patrimoine thérapeutique de ces hémiparasites auprès d'un large public.

Les objectifs consistent à évaluer la diversité spécifique des Loranthaceae dans les hautes terres de l'Ouest, à diffuser auprès des populations locales victimes des dégâts causés par ces hémiparasites les méthodes de contrôle les plus efficaces et accessibles - tout en respectant les normes de gestion durable de la ressource et en tenant compte de leurs potentialités thérapeutiques dans la zone - et à dresser un état des lieux des connaissances endogènes ou du savoir traditionnel maintenus jusqu'à aujourd'hui. La prise en compte intégrée de l'ensemble de ces aspects pourrait permettre de bâtir un schéma directeur dans le dessein d'une gestion plus durable du gui d'Afrique. 


\section{Approche méthodologique}

Ce travail repose principalement sur des observations de terrain et sur l'utilisation des outils qualitatifs et quantitatifs de la méthode active de recherche et de planification participative (Marpp). Cette dernière se fonde sur le profil historique (rites, interdits et connaissances ancestrales), les entretiens individuels dans le cadre d'enquêtes ethnobotaniques structurées individuelles et semistructurées de groupe, menés auprès de quelques personnes-ressources, notamment les tradithérapeutes, des personnes âgées d'au moins 50 ans, par la méthode d'interviews directes, au moyen de fiches d'enquête.

\section{Site de l'étude}

Le groupement Bafou (figure 1) s'étend entre 1400 à 2700 m d'altitude, sur une aire atteignant $170 \mathrm{~km}^{2}$. Il a été subdivisé en trois grandes zones écologiques et neuf localités ont été choisies pour être prospectées. Ont été retenues : dans la partie nord située entre 2000 et $2200 \mathrm{~m}$, les localités de Femmock (2,3 km²), Sankia (1,8 km²) et Nzi'i ndo $\left(2,1 \mathrm{~km}^{2}\right)$; sur le plateau basaltique situé entre 1500 et $1900 \mathrm{~m}$, les localités de Sessa $\left(2,8 \mathrm{~km}^{2}\right)$, Lefé $\left(0,98 \mathrm{~km}^{2}\right)$ et Zemla $\left(1,2 \mathrm{~km}^{2}\right)$; au sud, dans les enclaves granitiques (1 $400 \mathrm{~m}$ ), les localités de Fokamezou $\left(1,75 \mathrm{~km}^{2}\right)$, Batsinla $\left(0,89 \mathrm{~km}^{2}\right)$ et Ghonto $\left(3,7 \mathrm{~km}^{2}\right)$.

\section{Enquêtes ethnobotaniques}

Des enquêtes se basant sur des interrogations directes portant sur les usages des Loranthaceae dans la pharmacopée traditionnelle ont été conduites de juillet à septembre 2004 dans les neuf localités citées ci-dessus. Notre appartenance à ce groupement a permis de communiquer et de réaliser sans difficulté cette enquête.

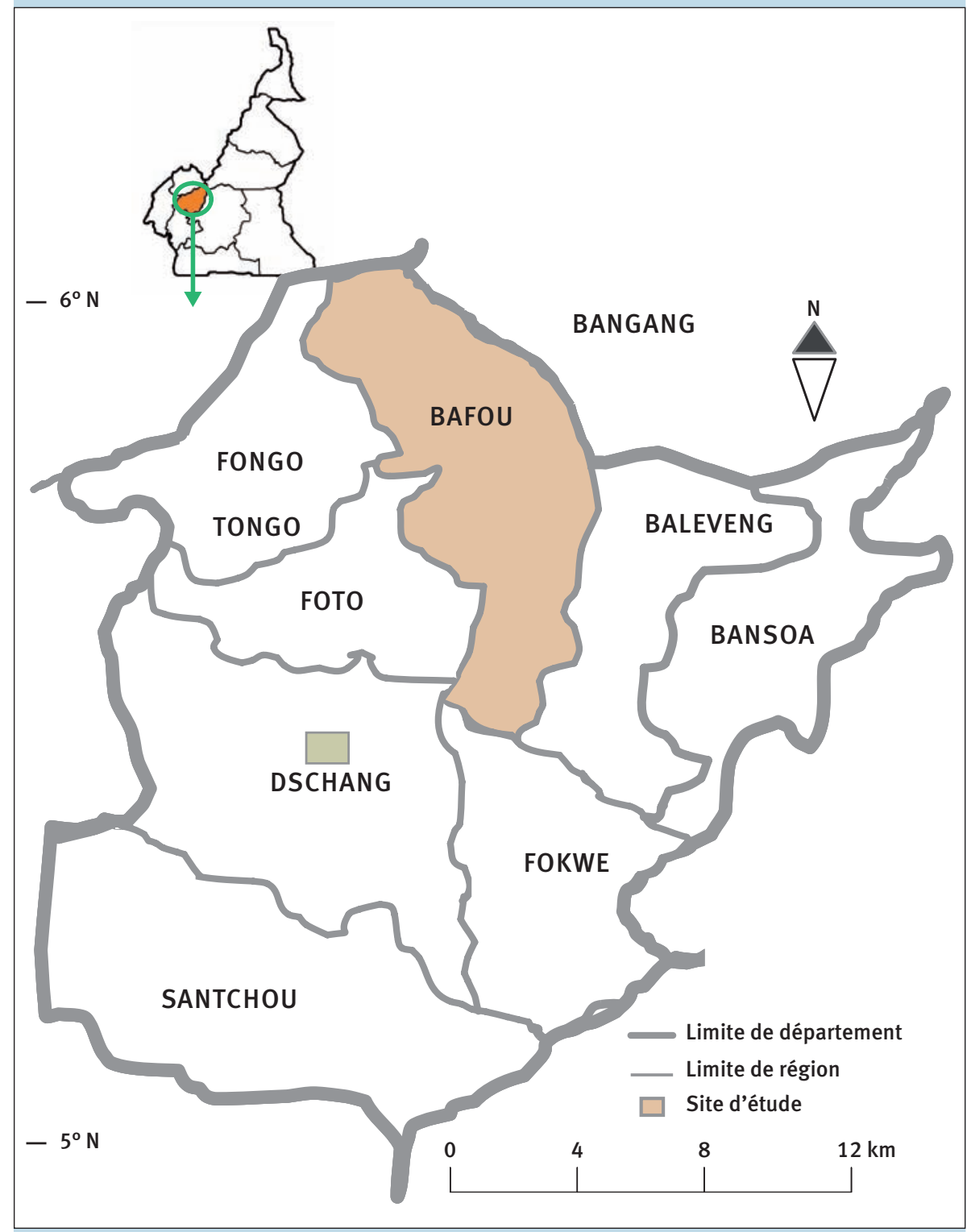

Figure 1.

Site d'étude et localisation du groupement Bafou dans les hautes terres de l'Ouest du Cameroun.

Des fiches d'enquête ont été élaborées dans le but de recueillir notamment des informations sur les parasites, les espèces hôtes parasitées, le parasite le plus fréquent, les hôtes les plus vulnérables, les parties de la plante utilisées et leurs techniques de récolte, les méthodes de préparation, les maladies guéries, la posologie, les autres usages et les méthodes de gestion locale de la ressource.

Ces informations sur les connaissances locales ont été recueillies auprès des tradithérapeutes réputés pour guérir ou soulager des maux avec le gui d'Afrique, des vieillards bénéficiant d'informations personnelles et/ou d'expériences vécues, et des femmes âgées de plus de 40 ans disposant de connaissances sur les usages et autres savoir-faire ancestraux de ces plantes. Toutes ces fiches conçues à l'endroit d'un public adulte tiennent compte des normes andragogiques relatives à l'éducation des adultes, nécessitant l'utilisation de termes ou d'un vocabulaire très familiers et moins scientifiques. 
Tableau I.

Importance des Loranthaceae dans la pharmacopée traditionnelle du groupement Bafou.

Hémiparasites

Agelanthus brunneus (Engl.) Balle \& N. Hallé

Globimetula braunii (Engl.) Tiegh.

Globimetula dinklagei (Engl.) Polhill \& Wiens

Phragmentera capitata (Spreng.) S. Balle

Tapinanthus bangwensis (Engl. \& K. Krause) Denser

Tapinanthus globiferus (A. Rich.) Tiegh.
Usages thérapeutiques

Ménopause, fétichisme, rhumatismes, courbatures, toux et infections pulmonaires

Diabète, blessures sorcières (infection pulmonaires occasionnées par vampirisme), hypertension artérielle, troubles cardiaques, anxiété et insomnie

Activités glandulaires, rythme cardiaque et digestion Abcès, furoncles, aphtes, courbatures et goutte Coups du sort, aphtes, goutte, rhumatismes, troubles cardiaques

Infections et congestion des voies urinaires, hypertension

\section{Résultats}

\section{Collecte de données}

Les hémiparasites inventoriés ont été identifiés sur le terrain, notamment dans les agroforêts et jardins de case. Des échantillons ont été récoltés et leur identification a été validée à l'Herbier national de Yaoundé. Les récoltes d'échantillons consistaient en des prélèvements sur l'arbre hôte au moyen d'un sécateur. Toutes les espèces hôtes ont été répertoriées et identifiées. Le degré de préférence de chaque parasite a été évalué en fonction des hôtes et des maladies. Les informations relatives à une gestion plus rationnelle ont été soigneusement notées. Enfin, quelques infestations artificielles ont été réalisées sur le manguier (Mangifera indica) afin d'évaluer l'aptitude du parasite à se développer sur cet hôte relativement peu commun, cependant très apprécié non seulement des tradithérapeutes, mais également des populations locales et autres chercheurs botanistes, biochimistes, chimistes, ethnobotanistes, etc., qui prélèvent et exploitent non seulement les organes à des fins de manipulations scientifiques, mais s'intéressent aussi à l'impact de l'exploitation sur la survie et la pérennité de l'espèce.

\section{Les espèces de Loranthaceae prospectées dans le groupement Bafou}

Parmi les 25 espèces de Loranthaceae signalées par BALLE (1986) au Cameroun, six ont été recensées et identifiées dans le groupement Bafou : Agelanthus brunneus (Engl.) Balle \& N. Hallé, Globimetula braunii (Engl.) Tiegh., Globimetula dinklagei (Engl.) Polhill \& Wiens, Phragmantera capitata (Spreng) S. Balle, Tapinanthus globiferus (A. Rich.) Tiegh., Tapinanthus bangwensis (Engl. \& K. Krause) Denser (JIOFACK, 2005 ; JIOFACK et al., 2007). L'identification des espèces sur le terrain n'est pas toujours aisée en raison des variations morphologiques imputables à leur phénologie de la reproduction. Les principaux caractères morphologiques fiables concernant les fleurs, les fruits et les feuilles ne perdurent pas sur la plante tout au long de l'année. Aussi, les paysans bafou regroupent toutes les espèces de Loranthaceae sous l'unique appellation de «Tsapla » en langue yemba, qui signifie plante cancérigène, ou " plante destructrice ou satanique ». Quant à l'ethnobotanique du gui, les différentes utilisations traditionnelles sont rassemblées dans le tableau I.

\section{Principaux hôtes des Loranthaceae}

Les Loranthaceae du groupement Bafou parasitent divers hôtes dont les principaux sont répertoriés dans le tableau II. On observe une prédominance de fruitiers comestibles qui sont très vulnérables à ces attaques parasitaires. Toutefois, la notion de spécificité reste un facteur capital dans l'orientation du choix du parasite à récolter par le tradithérapeute. Ce choix est très souvent fonction des symptômes que présente le patient. De surcroît, chaque plante hôte héberge un parasite qui lui est propre, d'où la notion de spécificité du tradithérapeute.

Au regard du tableau II, plus de $80 \%$ des tradithérapeutes utilisent ou sollicitent le gui du manguier (Mangifera indica, Anacardiaceae) et $60 \%$ celui du safoutier (Dacryodes edulis, Burseraceae). Également, près de $70 \%$ des tradithérapeutes recourent au gui présent sur Eucalyptus spp. (Myrtaceae).

Par ailleurs, le tableau fait état de neuf espèces d'arbres fruitiers très utilisées par les paysans. Cellesci sont regroupées dans sept familles végétales. On remarque en outre une certaine spécificité dans le choix de l'hôte. C'est notamment le cas du genre Globimetula, qui parasite Man- 
Tableau II.

Répartition des hémiparasites et de leurs hôtes en fonction de l'importance thérapeutique.

\begin{tabular}{|c|c|c|c|}
\hline Hôte vulnérable & Famille & Hémiparasites & $\begin{array}{c}\text { Importance } \\
\text { thérapeutique (\%) }\end{array}$ \\
\hline Mangifera indica & Anacardiaceae & $\begin{array}{l}\text { Tapinanthus sp. } \\
\text { Globimetula sp. }\end{array}$ & $80+$ \\
\hline Dacryodes edulis & Burseraceae & $\begin{array}{l}\text { Globimetula braunii } \\
\text { Agelanthus brunneus }\end{array}$ & $60+$ \\
\hline Canarium schweinfurthii & & Agelanthus brunneus & 20 \\
\hline Persea americana & Lauraceae & $\begin{array}{l}\text { Agelanthus brunneus } \\
\text { Phragmantera capitata } \\
\text { Tapinanthus bangwensis } \\
\text { Tapinanthus globiferus }\end{array}$ & 40 \\
\hline Psidium guajava & Myrtaceae & Agelanthus brunneus & 36 \\
\hline Eucalyptus sp. & & $\begin{array}{l}\text { Globimetula braunii } \\
\text { Globimetula dinklagei } \\
\text { Phragmantera capitata }\end{array}$ & $70+$ \\
\hline Coffea arabica & Rubiaceae & $\begin{array}{l}\text { Globimetula braunii } \\
\text { Tapinanthus bangwensis }\end{array}$ & 45 \\
\hline Citrus spp. & Rutaceae & $\begin{array}{l}\text { Agelanthus brunneus } \\
\text { Globimetula braunii } \\
\text { Phragmantera capitata }\end{array}$ & 30 \\
\hline Cola nitida & Sterculiaceae & $\begin{array}{l}\text { Phragmantera capitata } \\
\text { Tapinanthus bangwensis }\end{array}$ & 33 \\
\hline
\end{tabular}

gifera indica, Dacryodes edulis, Eucalyptus spp., Coffea arabica et Citrus spp. L'espèce Agelanthus brunneus infeste abondamment les plantes appartenant à la famille des Burseraceae, Lauraceae, Myrtaceae et Rutaceae. Le genre Tapinanthus est polyphyte mais exclut les Burseraceae,
Myrtaceae et Rutaceae. Ces informations restent partielles et découlent du présent travail. En raison des aires de prospection différentes de la forêt, la majorité des espèces parasitées sont des plantes introduites, très vulnérables aux attaques parasitaires. Une étude complémentaire de la croissance sur hôte spécifique permettrait de mieux préciser le lien étroit existant entre le parasite et sa préférence pour un hôte précis. Des résultats similaires ont été obtenus dans la région de Yaoundé au Cameroun sur Persea americana (lauracée) par SonkÉ et al. (2000). 


\section{Germination du gui sur hôte spécifique}

Le manguier (Mangifera indica, Anacardiaceae) est une plante dont les différentes parties interviennent efficacement dans la préparation de nombreux médicaments traditionnels. Le gui prélevé sur cet arbre serait également très riche en substances actives médicinales. La présence du parasite sur cet hôte, qui suscite l'intérêt des tradithérapeutes, reste cependant très rare dans la région. Selon les tradithérapeutes et quelques personnes-ressources, les parasites prélevés sur cet hôte soulageraient des patients souffrant de maladies congénitales et même virales. Afin d'étudier le pouvoir de dissémination du gui sur cet hôte spécifique très prisé, des essais de germination artificielle des graines de Globimetula dinklagei ont été conduits sur pied et des observations ont été réalisées.

\section{Gestion endogène des populations d'arbres hôtes}

Lors du prélèvement des parties utilisées dans la préparation des phytomédicaments et des rites respectés par les tradithérapeutes, les haustoria (organes d'ancrage et de fixation dans les tissus de l'arbre hôte, par lesquels le parasite prélève l'eau et les sels minéraux) de ces hémiparasites sont laissés intacts, ce qui permet au parasite de se régénérer plus facilement. Par contre, les paysans procèdent généralement à une coupe entière et exagérée des branches parasitées, ce qui compromet non seulement la productivité de la plante hôte, mais aussi l'abondance spécifique du parasite. Certains assimilent le gui au " cancer » à cause de la croissance anastomosée de son haustorium, dans les vaisseaux de liber où il prélève la sève brute. Cette faculté d'installation occasionne la formation de boursouflures ou bourrelets qui constituent une porte d'entrée ultérieure à de nombreux microorganismes pathogènes. Le paysan n'a alors plus pour solution que la suppression de la branche portant le parasite.

\section{Discussion}

Les loranthacées utilisées ici sont tour à tour sollicitées et participent soit isolément, soit en association dans la préparation d'une ou de plusieurs potions médicales. Les organes végétaux utilisés par les tradithérapeutes sont les feuilles, les écorces de tiges, les fleurs et les fruits. Les traitements se font par breuvage, friction, gargarisme et lavement. Les données du tableau I montrent l'implication et l'importance du gui dans la pharmacopée traditionnelle du groupement Bafou, les symptômes de plusieurs maladies étant combattus grâce à ces hémiparasites. Les feuilles des espèces de Tapinanthus spp. contiennent des polyphénols ayant des propriétés hypotensives et des substances actives telles que les lectines et viscotoxines, qui ont une action immunostimulante (TSABANG et al., 2005). Des effets magico-religieux ont été signalés lors des entretiens avec les tradithérapeutes et les vieillards, s'agissant notamment des risques d'avortement des femmes enceintes et de la perte de l'aptitude à la marche de nouveau-nés dans des concessions où le gui est utilisé comme bois de chauffe. Ces résultats sont similaires à ceux évoqués par AdjANOHOUN (2001) sur les plantes magiques, où il signale que les Tapinanthus s'emploient dans des cas d'exorcisme et de mauvais sort ; il ajoute que celui qui utilise les Tapinanthus poussant sur Calotropis procera (Asclepiadaceae) est protégé de toutes les maladies, y compris virales. Face à ce constat, les tradithérapeutes bafou signalent à leur tour que toute espèce de gui prélevée sur Mangifera indica donnerait une sorte de pouvoir. Le gui soulagerait également les patients souffrant de maladies congénitales et/ou héréditaires.

Une affinité du gui pour les fruitiers a été notée et s'expliquerait par le fait que les oiseaux disséminateurs (Pogoniulus chrysonocus) et pollinisateurs (Nectarina senegalensis et $N$. pulchella) sont les principaux vecteurs à l'occasion de leurs passages

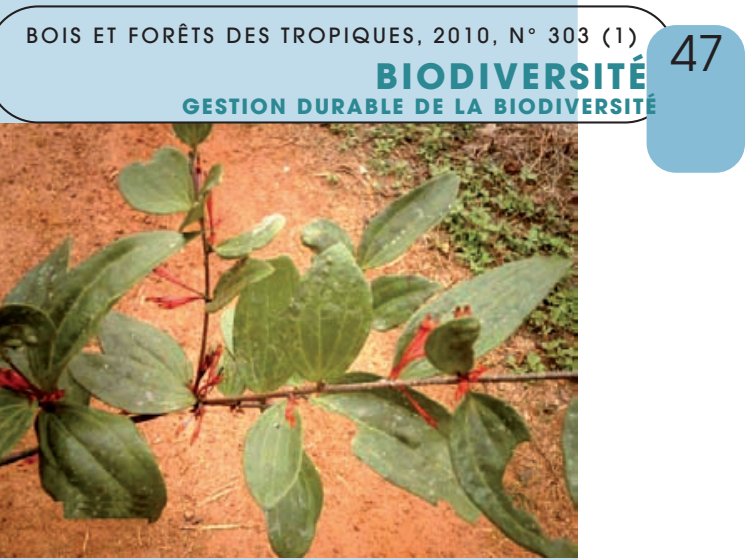

Agelanthus brunneus (rameau florifère). Photo T. Jiofack.

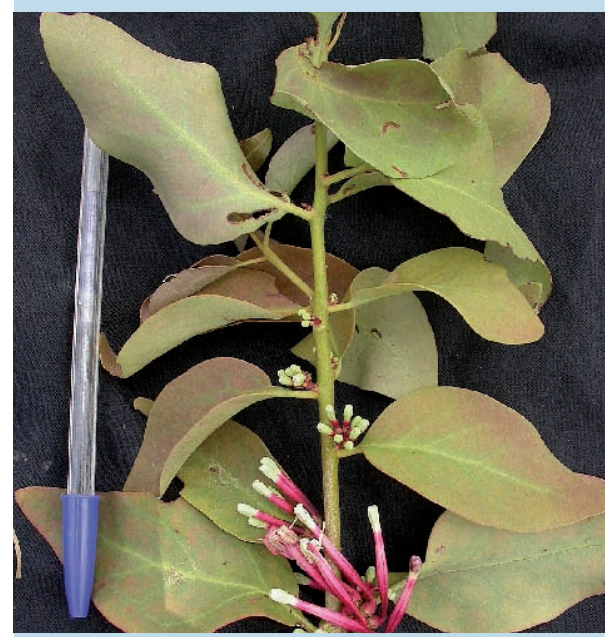

Tapinanthus bangwensis (jeune rameau végétatif avec début de florogenèse). Photo T. Jiofack.

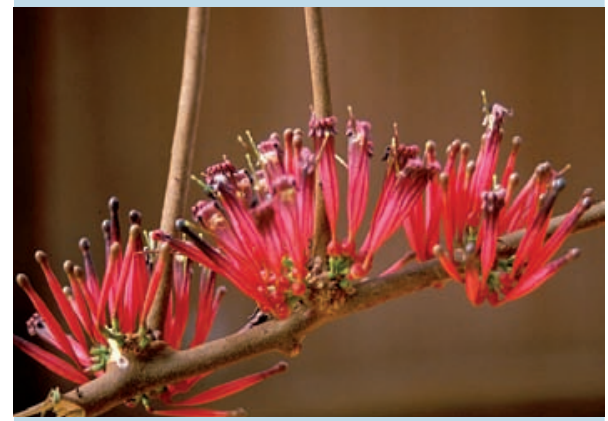

Tapinanthus bangwensis (inflorescence mature). Photo G. Glatzel.

dans les arbres hôtes. Les données du tableau II s'expliqueraient par le fait que des principes actifs que possèdent ces plantes (eucalyptus), notamment l'eucalyptuine, sont très opérants dans le traitement des toux et la décongestion des voies respiratoires. Ce constat est à l'origine du concept de priorisation des hôtes spécifiques aux hémiparasites dans le processus de gestion durable des ressources biologiques. 
Le cycle biologique du gui euro-

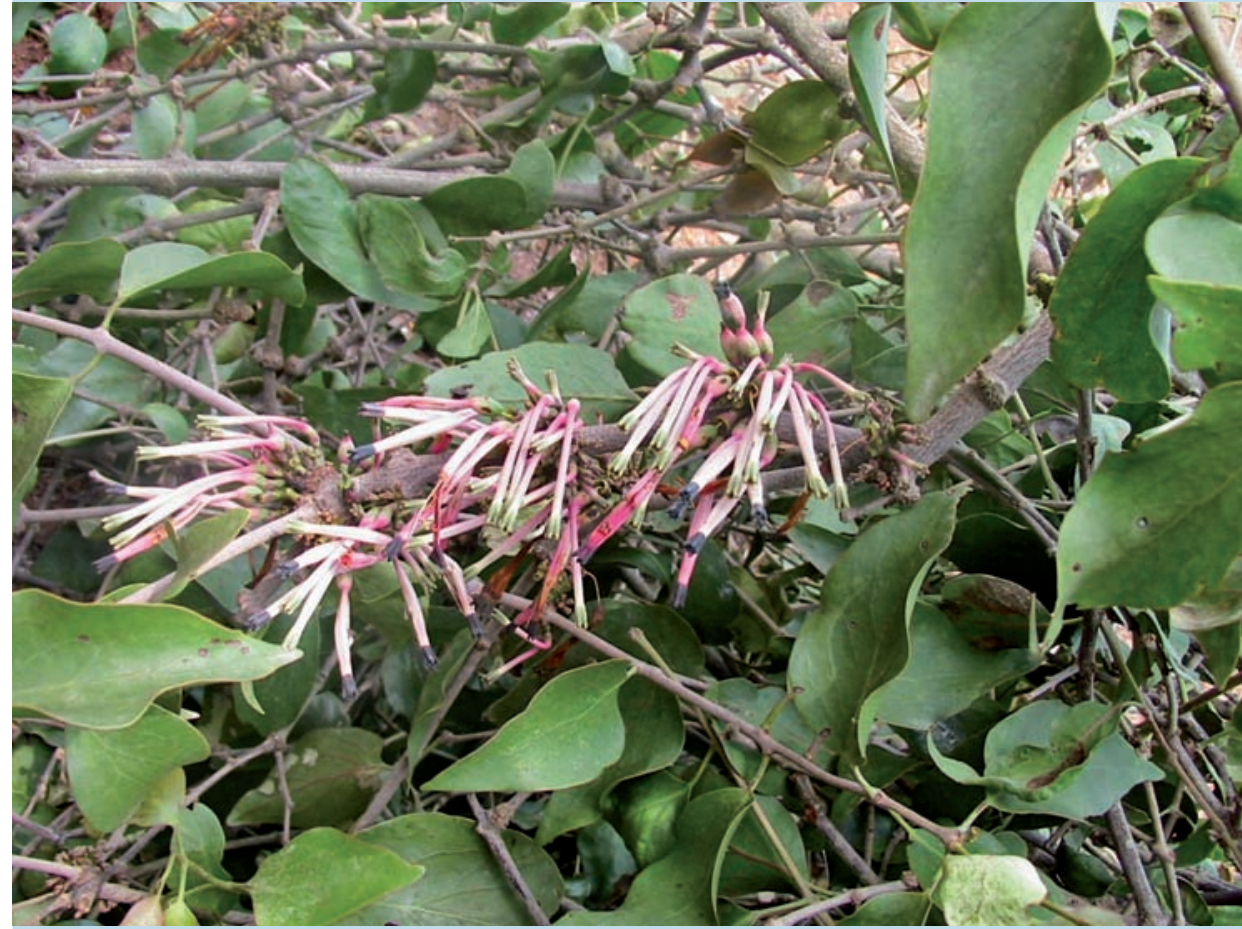

Tapinanthus bangwensis (rameau florifère). Photo T. Jiofack.

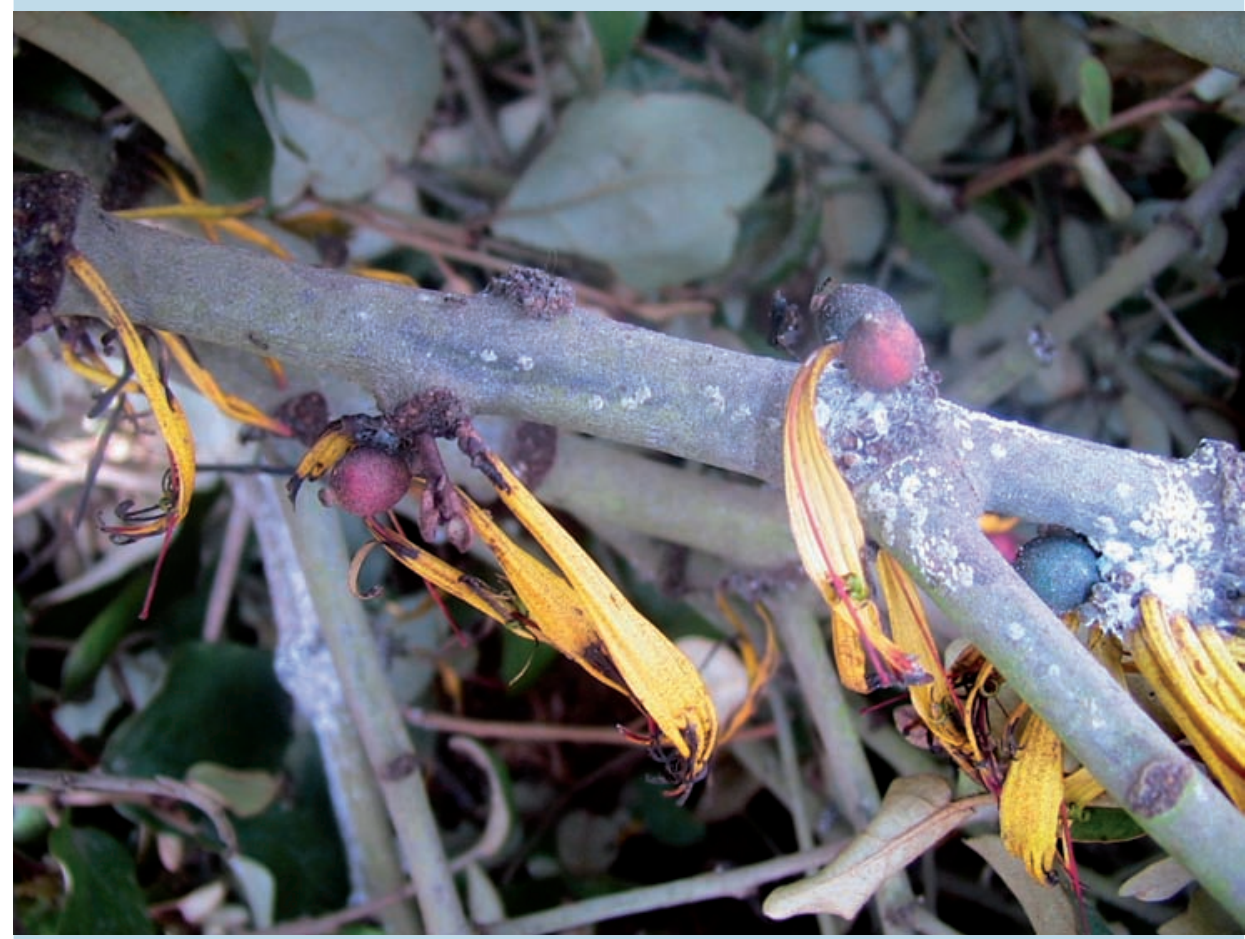

Phragmantera capitata (coexistence de fleurs et fruits matures sur une même branche).

Photo T. Jiofack. péen (Viscum album) dure trois ans (Boussım et al., 2004). En revanche, en Afrique, du début de la germination jusqu'à l'étape de la feuillaison, moins de 21 jours s'écouleraient, témoignant d'une croissance très rapide. Des essais de germination conduits sur Tapinanthus par Boussim et al. (1993 a et b) ont montré que la contamination est possible sur tout organe vivant ou non, des surfaces inertes et même des plaques de verre. Mais la fixation n'est réalisable que sur un hôte vivant. Dans le cas du développement du gui sur le manguier, les essais réalisés ont ainsi montré que la germination est possible mais que la fixation ne l'était pas. SALLÉ et ABER (1986) expliquent l'absence de développement sur le manguier par un manque de raccordement vasculaire entre les tissus de l'hôte et l'haustorium du parasite. Ils invoquent également la présence d'une barrière chimique constituée de tanins, et qui est mise en place au niveau du cambium de l'hôte, en réponse à la pénétration de l'haustorium du parasite.

Pour ce qui est de la gestion durable proprement dite de ces hémiparasites, vu les difficultés inhérentes au recours à une méthode de lutte efficace et l'intérêt écologique pour l'écosystème forestier, ces Loranthaceae sont considérées comme un élément constitutif de l'écosystème à gérer. Elles causent cependant d'importants dégâts sur les récoltes et compromettent la production fruitière sur des arbres fruitiers dont la récolte est principalement destinée à l'autoconsommation ou à la vente. Malgré leur caractère nuisible, ces hémiparasites possèdent néanmoins des propriétés thérapeutiques indéniables. II se pose ainsi un réel problème de conservation d'une ressource présentant des conflits d'intérêts. Les paysans souhaiteraient en effet éradiquer ces 
parasites qui constituent un fléau pour leurs arbres fruitiers, et les tradithérapeutes voudraient les conserver pour soulager leurs patients. Face à ce dilemme, une gestion durable de cette ressource médicinale pourrait s'appuyer sur :

- la suppression contrôlée des branches parasitées sur l'hôte, notamment pendant la période de repos de ce dernier (période de nonfructification et de non-floraison) ;

- la priorisation des hôtes spécifiques comme le manguier sur qui le parasite demeure très rare ;

- la culture artificielle de l'hémiparasite sur des hôtes spécifiques, puis le contrôle du vecteur (Pogoniulus chrysonochus) responsable des propagations incontrôlées.

Les résultats des études conduites par JIOFACK (2005) et JIOFACK et al. (2007) dans la région montrent que le contrôle par la suppression de la branche parasitée et de l'haustorium est assuré par plus de $80 \%$ des paysans locaux, alors que l'utilisation de la méthode préventive de contrôle du vecteur n'est appliquée que par moins de $5 \%$ des personnes interviewées. En effet, les populations locales connaissent parfaitement cet oiseau qu'elles combattent, pour les uns régulièrement à l'aide des frondes fourchues. Le faible pourcentage d'utilisation de cette technique montre qu'elle est pénible et contraignante. Boussim et al. (2004), SAlLÉ et al. (1993) et Boussım (2002) démontrent également que la coupe constitue la méthode curative la plus simple, et qu'elle est efficiente si la branche hôte est coupée à quelque centimètres en dessous du point de fixation du parasite. Bien que ces méthodes soient moins concluantes, elles sont désespérément utilisées par les paysans du groupement mais les plus efficaces, recensées par SALLÉ (2002, 2003), associent entre autres la lutte biologique, la lutte chimique, la résistance génétique et la lutte intégrée.

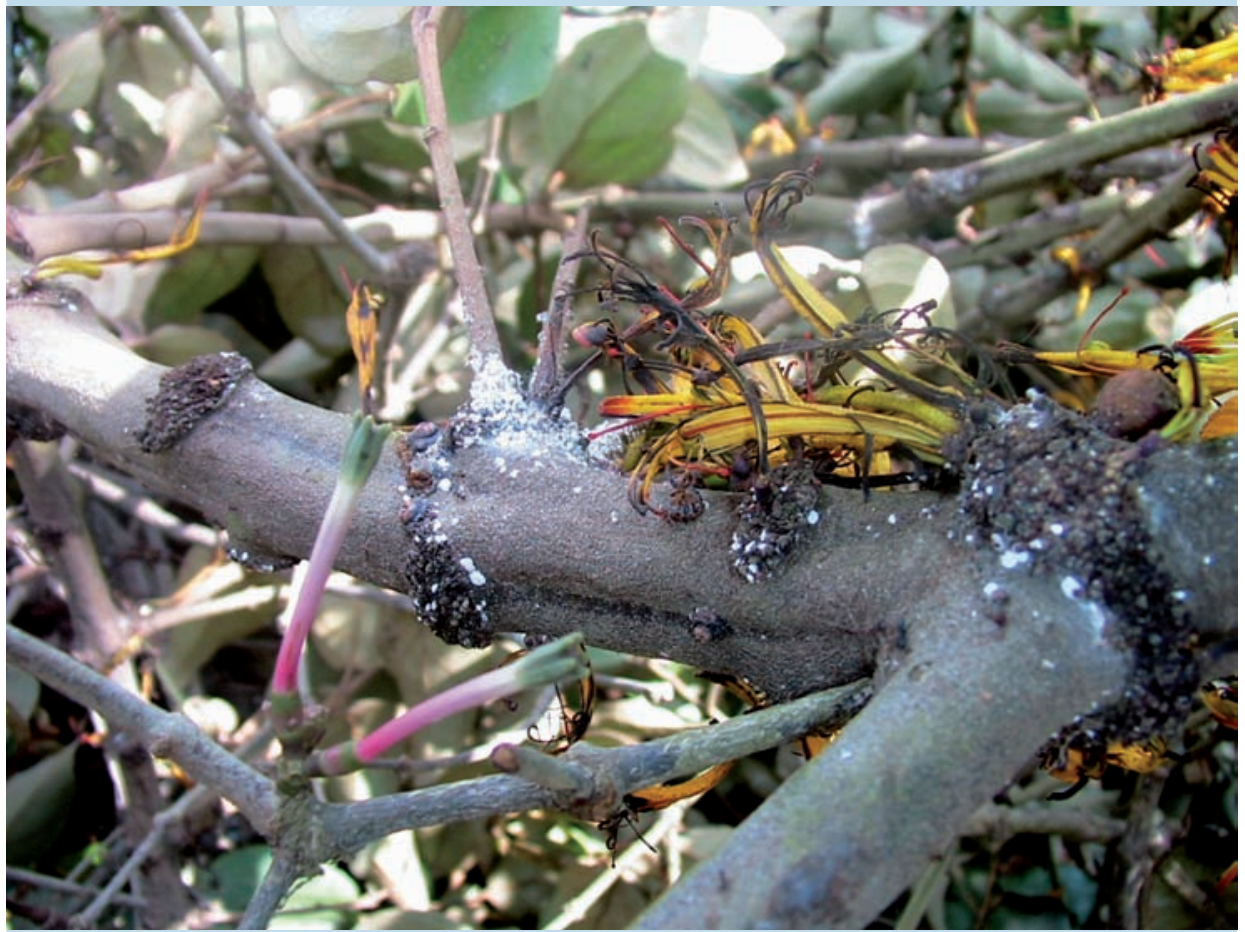

Phénomène d'hyperparasitisme de premier degré (parasitisme d'une loranthacée (Phragmantera capitata, à fleurs jaunes) par une autre loranthacée (Tapinanthus bangwensis, à fleurs rose blanchâtre). Photo T. Jiofack.

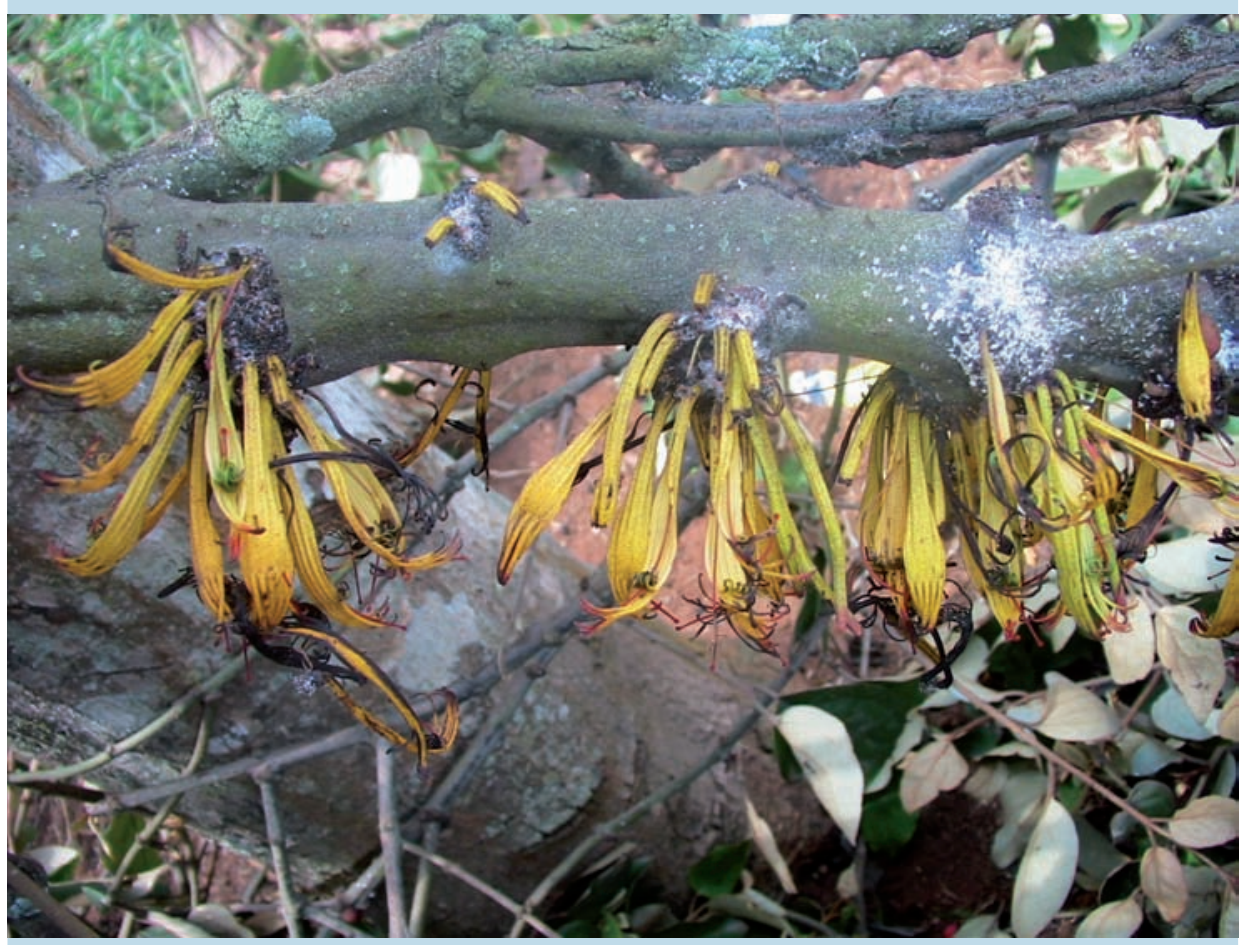

Phragmantera capitata (vieux rameau florifère). Photo T. Jiofack. 


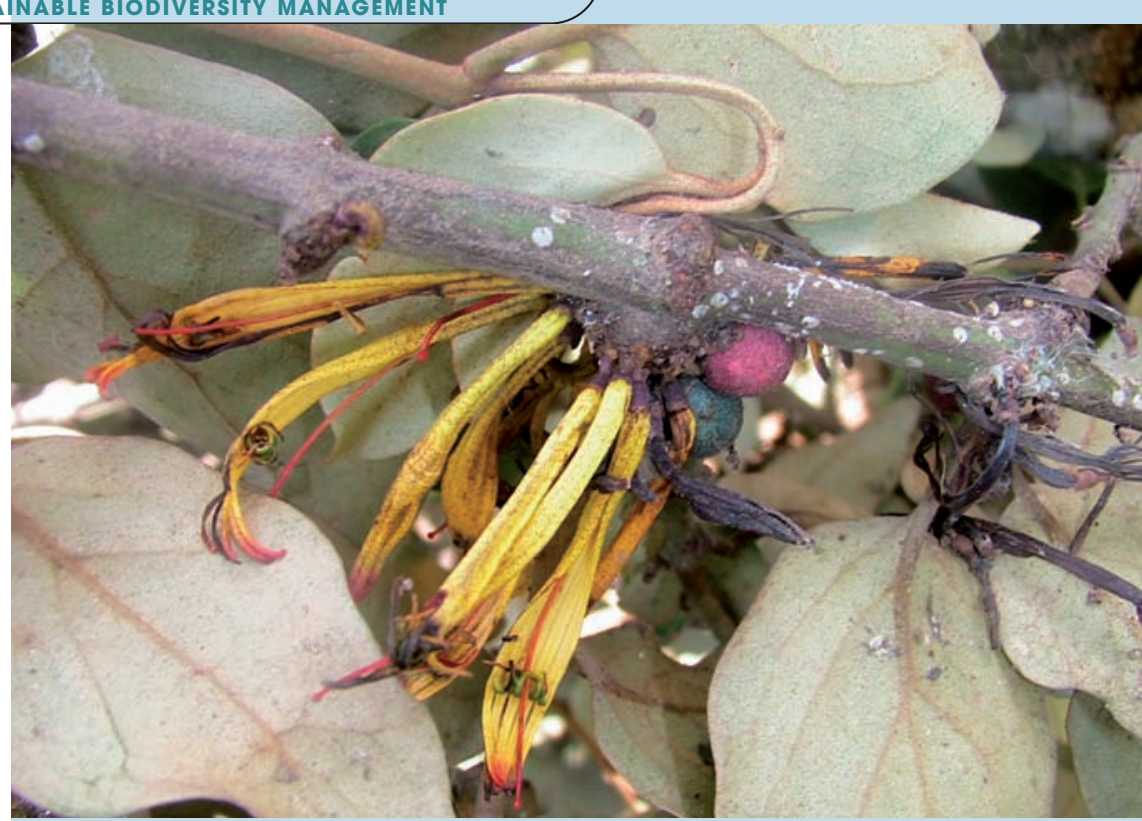

Phragmantera capitata : rameau présentant des fleurs matures (jaunes), dégénérées (noires), des fruits matures (rouges) et immatures (verts) et la face inférieure argentée des feuilles). Photo T. Jiofack, 2008.
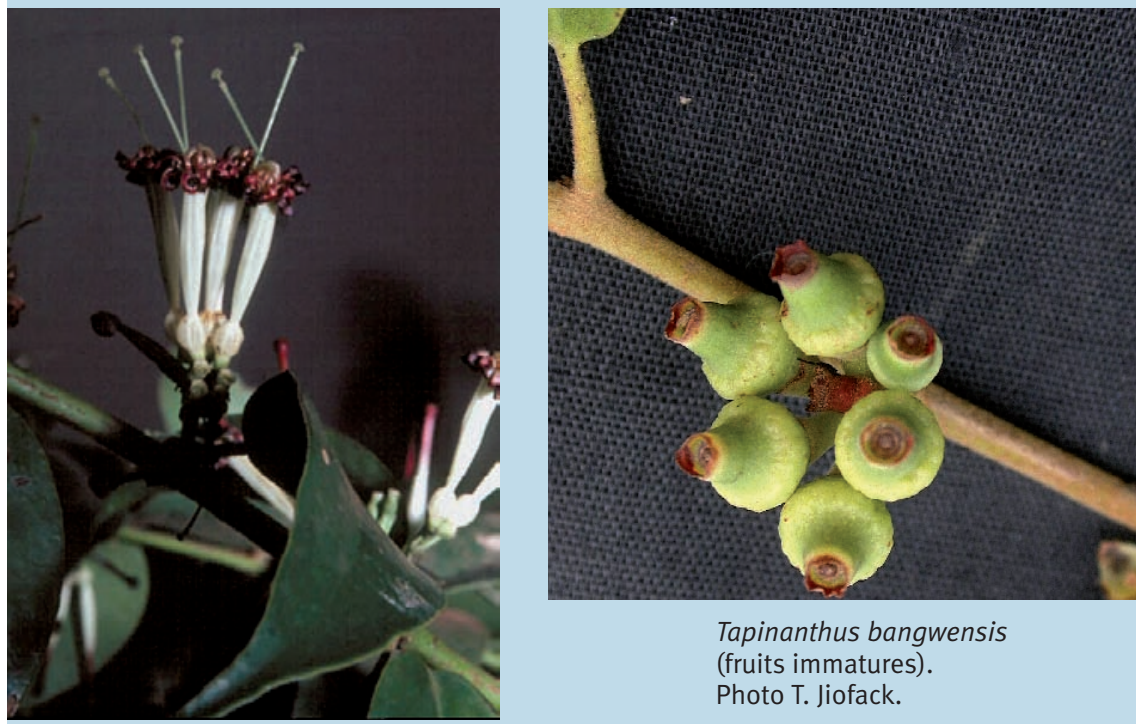

Tapinanthus bangwensis (fruits immatures) Photo T. Jiofack.

Globimetula dinklagei

(bouton floral).

Photo G. Glatzel.

En attendant que ces nouvelles méthodes (coûteuses et très sélectives) qui respectent les normes de gestion durable soient applicables dans le continent africain en général et au Cameroun en particulier, il serait judicieux d'orienter les paysans vers la méthode la plus simple, mais malheureusement oubliée, qui consiste à éviter de laisser fleurir et fructifier la Loranthaceae sur l'hôte, notamment pendant la période de repos de ce dernier. Cela semble irréaliste, mais pourtant appliqué par les paysans, surtout ceux qui vivent essentiellement de la vente des produits issus de leurs arbres fruitiers.

Si les coupes trop destructrices restent néfastes pour la conservation de la biodiversité végétale (hôte et hémiparasite), l'arrachage pratiqué par les tradithérapeutes n'endommage pas le système d'absorption du parasite qui a la faculté de se régénérer très facilement.

\section{Conclusion}

Le groupement Bafou recèle six espèces de Loranthaceae intervenant soit isolément, soit en association avec d'autres plantes pour soigner ou soulager plusieurs maladies: Agelanthus brunneus, Globimetula braunii, Globimetula dinklagei, Phragmantera capitata, Tapinanthus globiferus et Tapinanthus bangwensis. Ces hémiparasites infestent les végétaux sur la totalité de leur aire de répartition et s'étendent dans les localités limitrophes. Leur extension est favorisée non seulement par l'abondance de fruitiers sur qui ils prolifèrent aisément mais aussi par leur caractère polyphyte.

Bien que ces hémiparasites soient utilisés comme palliatifs dans le patrimoine thérapeutique traditionnel, des études complémentaires restent nécessaires pour isoler les principes actifs, et surtout évaluer leur toxicité. Une culture adéquate de ces hémiparasites sur hôtes spécifiques permettrait d'étendre leurs utilisations dans les domaines de la médecine traditionnelle et de la recherche médicale. Cette étude encore incomplète vient en outre étayer le principe selon lequel le contrôle de ces hémiparasites pourrait contribuer à la conservation de l'hôte en particulier et des populations d'arbres en général dans le groupement Bafou. Toutefois, les différentes méthodes de contrôle du parasite n'obéissent pas nécessairement à une gestion durable de la ressource, sauf si les approches de gestion évoquées plus haut sont prises en considération ; ainsi, le paysan conserverait mieux l'espèce et la diversité des populations d'arbres de la région. 


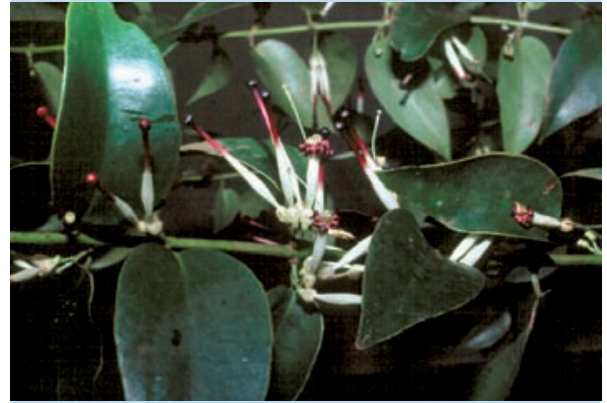

Globimetula dinklagei (rameau florifère et végétatif). Photo G. Glatzel.

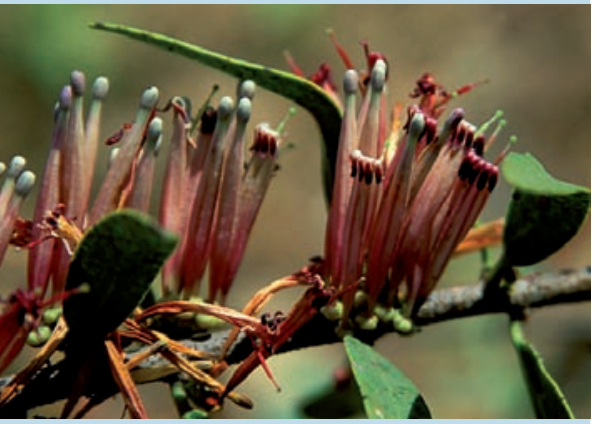

Tapinanthus globiferus

(rameau florifère).

Photo G. Glatzel.

\section{Remerciements}

Sont remerciées toutes les personnes qui ont contribué à la réalisation de cette étude : les tradithérapeutes bafou contactés, la famille Téfokou Joseph (doyen) pour son soutien financier, le personnel de l'Herbier national de Yaoundé pour l'identification et la confirmation des échantillons, ainsi que le Département de foresterie de l'Université de Dschang et le Laboratoire de botanique et d'écologie de l'Université de Yaoundé I pour leur appui logistique.

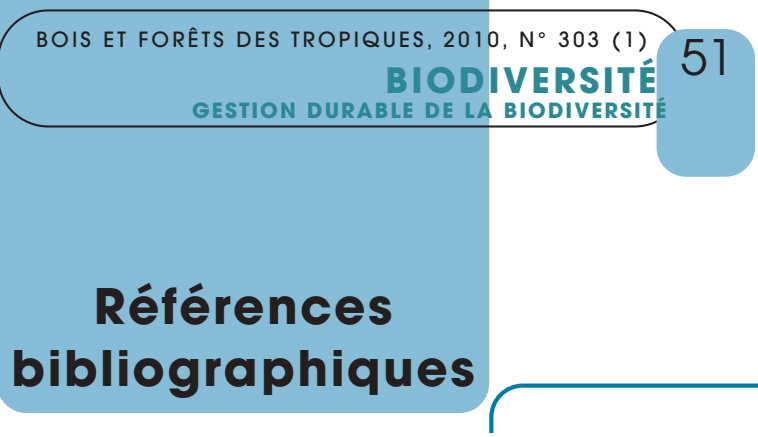

ADJANOHOUN E. J., 2001. La médecine traditionnelle au Bénin : recensement des savoir-faire traditionnels. Extrait du rapport Cenprebat-Cbdd. Revue de Médecines et Pharmacopées Africaines, 15 : 103-111.

BALLE S., 1986. Flore du Cameroun, 23. Loranthaceae (Éds B. Satabié et J.-F. Leroy). Yaoundé, Cameroun, Dgrst, 82 p.

BOUSSIM I. J., SALLÉ G., GUINKO S., 1993a. Tapinanthus parasite du karité au Burkina Faso. Première partie : Identification et distribution. Bois et Forêts des Tropiques, 238 : 45-52.

BOUSSIM I. J., SALLÉ G., GUINKO S., 1993b. Tapinanthus parasite du karité au Burkina Faso. Seconde partie : Phénologie, biologie et dégâts. Bois et Forêts des Tropiques, 238 : 53-65.
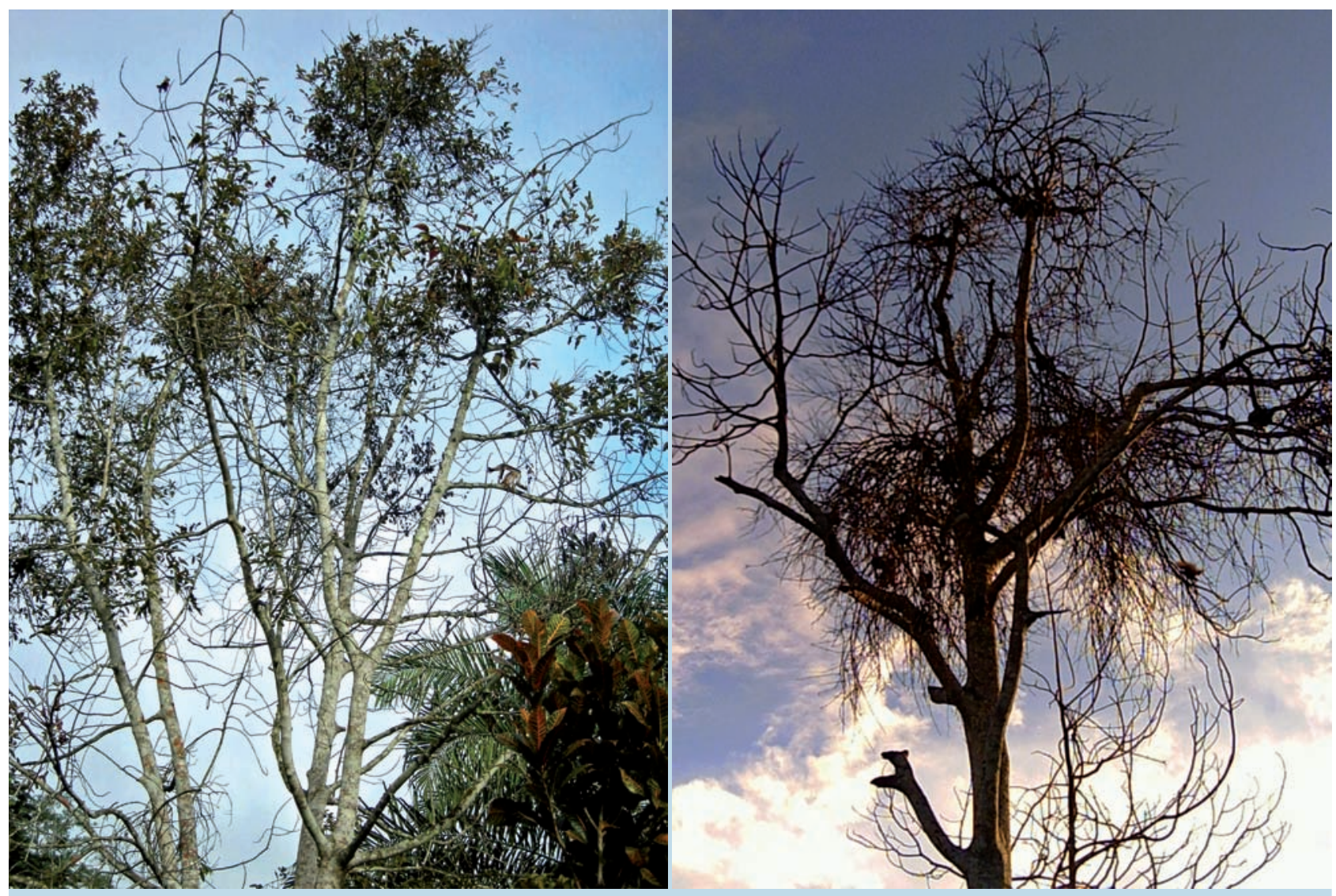
BOUSSIM I. J., 2002. Les phanérogames parasites du Burkina Faso : inventaire, taxonomie, écologie et quelques aspects de leur biologie. Cas particulier des Loranthaceae parasites du karité. Thèse de doctorat d’État ès sciences naturelles, Ouagadougou, Burkina Faso, 285 p.

BOUSSIM I. J., GUINKO S., TUQUET C., SALLÉ G., 2004. Mistletoes of the agroforestry parklands of Burkina Faso. Agroforestry Systems, 60 (1) : 39-49 (11).

DONGMO J.-L., TSALEFAC M., METANGMO G., TAZO E., 1990. Bafou : une grande chefferie de l'Ouest-Cameroun. Yaoundé, Cameroun, Éditions Ceper, 115 p.

JIOFACK T., 2005. Contribution à l'étude des Loranthaceae dans les Hautes Terres de l'Ouest Cameroun : cas du groupement Bafou. Mémoire de Dea, Université de Yaoundé I, Cameroun, 64 p.

JIOFACK T., KEMEUZE V., PINTA J., 2007. Les Loranthaceae dans la pharmacopée traditionnelle du groupement Bafou. Cameroon Journal of Ethnobotany, 2, $7 \mathrm{p}$.

LIEUTAGHI F., 1991. La plante compagne : pratique et imaginaire de la flore sauvage en Europe occidentale. Conservatoire et jardin botaniques de la ville de Genève, Suisse, 220 p.

POLHILL R., WIENS D. W., 1998. Mistletoes of Africa. Richmond, Royaume-Uni, Kew Publishing, 370 p.

RODZKO V., 2000. Abécédaire de phytothérapie. Éditions Interactive, $2 \mathrm{p}$.

SALLÉ G., FROCHOT H., ANDARY C., 1993. Le Gui. La Recherche, 24 : 1334-1342.

SALLÉ G., ABER M., 1986. Les phanérogames parasites : biologie et stratégies de lutte. Lettres Botaniques, 133 (3) : 235-263.

SALLÉ G., TUQUET C., RAYNALROQUES A., 1998. Biologie des phanérogames parasites. Comptes rendus des séances de la Société de biologie, 192 (1) : 9-36.
SALLÉ G., 2002. Rapport de la mission réalisée au Gabon du 8-17 avril 2002. Paris, France, Université Pierre et Marie Curie, Laboratoire de parasitologie végétale, $12 \mathrm{p}$.

SALLÉ G., 2003. Rapport de la mission réalisée au Gabon du 9-19 mai 2003. Paris, France, Université Pierre et Marie Curie, Laboratoire de parasitologie végétale, $14 \mathrm{p}$.

SONKÉ B., KENFACK D., TINDO R., 2000. Parasitisme de l'avocatier (Persea americana, Lauraceae) dans la région de Yaoundé (Cameroun). Fruits, 55 (5) : 325-331.

TSABANG N., NKONGMENECK B., NGUENANG G. M., FONGNZOSSIE F. E., KOUEKE P., BIYITI L., TAMZE V., FOKO J., MBITA MESSI J. C., ATANGANA NDONGO N. J., LEJOLY J., 2005. Médecine traditionnelle : une méthode d'identification des diabétiques et/ou hypertendus et des plantes pouvant améliorer leur état de santé. Cameroon Journal of Ethnobotany, 1 : 53-59. 